\title{
Evaluation of the rapid immunochromatographic ODK0501 assay for Streptococcus pneumoniae antigen detection with nasopharyngeal swabs: preliminary report
}

Shoji Suzuki, Naoki Nishimura*, Torahiko Jinta, Yasuhiko Yamano, Genta Ishikawa, Yutaka Tomishima, Noboru Uchiyama and Naohiko Chohnabayashi

\begin{abstract}
Background: Early identification and control of pathogenic bacteria are important in the treatment of pneumonia. Currently, two rapid antigen detection kits for pneumococcal pneumonia are available: one uses urine samples and the other, named RAPIRUN ${ }^{\circledR}$ S. pneumoniae, uses sputum samples. RAPIRUN ${ }^{\circledR}$ has shown high sensitivity with nasopharyngeal swab samples from pediatric patients. In this study, we investigated the performance of RAPIRUN® ${ }^{\circledR}$ with nasopharyngeal swabs from adult patients.

Methods: All adult patients diagnosed with pneumonia from November 2011 to April 2012 in St. Luke's International hospital were included in this cross-sectional study. Single sputum, nasopharyngeal swab, and urine samples obtained from patients were investigated using a rapid antigen detection kit. Sputum and blood cultures were also evaluated. We compared the characteristics of pneumococcal pneumonia patients diagnosed using RAPIRUN with a nasopharyngeal swab to those patients diagnosed using other methods. Sensitivity and specificity were also calculated.

Results: Seventeen out of 60 patients with pneumonia were diagnosed with pneumococcal pneumonia. In 4 out of the 17 cases, a positive test result was obtained using RAPIRUN with a nasopharyngeal swab. The sensitivity and specificity were 23.5 and $100 \%$, respectively.

Conclusion: RAPIRUN performed with nasopharyngeal swabs from adult patients exhibited lower sensitivity for the diagnosis of pneumococcal pneumonia than the other compared methods. The causative pathogen of pneumonia should be identified using not only sputum cultures or rapid antigen detection kits but also clinical features or gram staining of sputum.
\end{abstract}

Keywords: Immunochromatographic assay, Pneumonia, RAPIRUN S. pneumoniae, Nasopharyngeal swab

\footnotetext{
* Correspondence: nina@luke.ac.jp

Division of Pulmonary Medicine, St. Luke's International Hospital, 9-1,

Akashi-cho, Chuo City, Tokyo 104-8560, Japan
} 


\section{Background}

Pneumonia was the fourth most common cause of death worldwide in 2012 [1], and it is a critical illness especially for the elderly because of increase in its incidence and severity [2]. The most common causative pathogen of pneumonia is Streptococcus pneumoniae, which causes a serious form of pneumonia [3, 4]. Although pneumococcal vaccination is an efficient prevention strategy against pneumococcal pneumonia, the vaccine does not cover all serotypes of S. pneumoniae [5]. Thus, early identification of the pathogenic bacteria and appropriate initial treatment remain important to an optimal outcome.

Currently, two rapid detection kits for S. pneumoniae antigen are available: BinaxNOW ${ }^{\bullet}$ S. pneumoniae, which uses urine samples, and an immunochromatographic ODK0501 assay named RAPIRUN ${ }^{\circ}$ S. pneumoniae, which uses sputum and nasopharyngeal swab samples (hereafter referred to as BinaxNOW and RAPIRUN, respectively). It has been reported that the use of BinaxNOW with urine samples and RAPIRUN with sputum samples from adult pneumonia patients shows high efficiency in the diagnosis of pneumococcal pneumonia [6-10]. In addition, RAPIRUN performed with sputum samples shows higher sensitivity than BinaxNOW $[9,10]$. However, RAPIRUN requires good quality sputum to achieve higher sensitivity [11], and the sensitivity of RAPIRUN might be lower in the elderly, who have difficulty coughing up sputum.

RAPIRUN has also been found to exhibit high sensitivity for pneumococcal pneumonia when used with nasopharyngeal swabs from pediatric patients [12]. However, the efficiency of RAPIRUN with nasopharyngeal swabs from adult patients has not been reported. In this study, we investigated the performance of RAPIRUN with nasopharyngeal swabs for the diagnosis of pneumococcal pneumonia in adult patients.

\section{Methods}

\section{Patient population and diagnostic criteria}

The St. Luke's International Hospital Research Ethics Committee approved all aspects of this study (approval number 11-R127, approval date October 24, 2011). This was a cross-sectional study conducted at St. Luke's International Hospital, Tokyo, Japan, from November 2011 to April 2012. Written informed consent was obtained from 60 consecutive patients who were over 20 years old and were hospitalized with a diagnosis of pneumonia.

Pneumonia was defined as new infiltrates on a chest radiograph with symptoms indicating lower respiratory tract infection, such as cough, sputum, fever, or dyspnea.

\section{Sample collection and microbiological investigation}

Single sputum, urine, and nasopharyngeal swab samples were collected from the patients. Nasopharyngeal swab samples were collected by inserting swab into a nostril straight back along the floor of the nasal passage until reaching the posterior wall of the nasopharynx. Sputum and nasopharyngeal swab samples were analyzed using RAPIRUN $^{\bullet}$ (Otsuka Pharmaceutical Co., Ltd., Tokyo, Japan). We also analyzed the urine samples using the BinaxNOW $^{\circ}$ rapid antigen detection kit (Binax Inc., Scarborough, ME, USA). Sputum, nasopharyngeal, and blood cultures were also evaluated.

\section{Using RAPIRUN ${ }^{\circledR}$}

We used swabs to collect sputum and nasopharyngeal samples, and the extraction solutions were added to the test kit. Two lines indicated a positive result, i.e., the test and control, and a negative result showed only the control line. This test required approximately $25 \mathrm{~min}$ in total.

\section{Data collection}

In addition to bacterial information, we collected clinical data, such as those pertaining to age, sex, smoking habits, existing underlying diseases, residence, history of pneumococcal vaccination, prior antibiotic therapy, vital signs, medication, laboratory testing, and chest radiographs, at admission. We also calculated the Patient Outcomes Research Team (PORT) score.

\section{Diagnosis of pneumococcal pneumonia}

Sputum culture is the most commonly used reference standard for the detection of the causative pneumonia pathogens, but both the sensitivity and specificity are not sufficient [13, 14]. We determined the causative pneumonia pathogen using a combination of multiple diagnostic test results, that is the results for sputum culture, blood culture, and a rapid antigen detection kit (RAPIRUN with sputum and BinaxNOW with urine). A diagnosis of pneumococcal pneumonia was made if any test results showed evidence of S. pneumoniae.

\section{Analysis}

We compared the characteristics of patients with pneumococcal pneumonia with those of patients with pneumonia caused by other pathogens using Fisher's exact probability test or Mann-Whitney's $U$ test (two tailed, with a $p$ of $<0.05$ indicating a significant difference).

We also compared the patients with pneumococcal pneumonia diagnosed using RAPIRUN with a nasopharyngeal swab with those diagnosed using other methods. The sensitivity and specificity of RAPIRUN performed with a nasopharyngeal swab for diagnosing pneumococcal pneumonia were also calculated. 


\section{Results}

We collected samples from 60 consecutive inpatients with pneumonia. Due to anuria in cases of end-stage renal failure, urine samples were obtained from only 58 patients. The average age of all patients was 81 (28-96) years, and more than half of the patients were male $(58.3 \%)$ and smokers (55.0\%). Commonly, observed underlying diseases included diabetes mellitus, chronic obstructive pulmonary disease, and malignant neoplasm. Thirteen out of $60 \quad(21.7 \%)$ patients had received the 23-valent pneumococcal polysaccharide vaccine.

Seventeen out of $60(28.3 \%)$ patients had been diagnosed with pneumococcal pneumonia. For the 17 cases of pneumococcal pneumonia, 14 cases were positive using BinaxNOW, 12 cases were positive using RAPIRUN with sputum samples, 10 cases were positive for $S$. pneumoniae in the sputum culture, and one case was positive for $S$. pneumoniae in the blood culture. Table 1 lists the patients' characteristics. C-reactive protein was significantly higher in patients with pneumococcal pneumonia than in patients with pneumonia caused by other bacteria. The proportion of patients who received a pneumococcal vaccine was lower in patients with pneumococcal pneumonia, but this difference was not significant.
Results of RAPIRUN with nasopharyngeal swabs

Out of the 60 patients who participated in this study, only four patients showed positive test results when using RAPIRUN with nasopharyngeal swabs. The characteristics of these four patients and the results of the microbiological investigations are shown in Tables 2 and 3 , respectively. All four cases were diagnosed as pneumococcal pneumonia using BinaxNOW performed with a urine sample or RAPIRUN performed with a sputum sample. Interestingly, only two patients had positive nasopharyngeal culture for S. pneumoniae among four cases with positive result of RAPIRUN with a nasopharyngeal swab. There was an insufficient number of patients; however, the sensitivity and specificity of RAPIRUN with a nasopharyngeal swab were calculated as 23.5 and $100 \%$, respectively.

\section{Discussion}

We diagnosed 17 patients as having pneumococcal pneumonia, and only four of these patients showed positive results when using RAPIRUN with nasopharyngeal swabs, suggesting low sensitivity, although RAPIRUN with sputum samples previously showed high sensitivity [10]. Iwata et al. [12] reported that RAPIRUN exhibited high sensitivity $(33 / 50,66 \%)$ and specificity (55/55, $100 \%)$ when used with nasopharyngeal swabs in

Table 1 Characteristics of pneumonia patients

\begin{tabular}{|c|c|c|c|c|c|c|c|}
\hline \multirow[b]{2}{*}{ Age, years, median (range) } & \multicolumn{2}{|l|}{$\begin{array}{l}\text { Total } \\
(n=60)\end{array}$} & \multicolumn{2}{|c|}{$\begin{array}{l}\text { Pneumococcal pneumonia } \\
(n=17)\end{array}$} & \multicolumn{2}{|c|}{$\begin{array}{l}\text { Pneumonia caused by other } \\
\text { bacteria }(n=43)\end{array}$} & \multirow{2}{*}{$\begin{array}{l}P \\
0.45\end{array}$} \\
\hline & 81 & $(28-96)$ & 78 & $(28-96)$ & 82 & $(40-96)$ & \\
\hline Male sex, n (\%) & 35 & $(58.3)$ & 7 & $(41.2)$ & 28 & $(68.3)$ & 0.15 \\
\hline Nursing home residents, n (\%) & 8 & $(13.3)$ & 0 & 0 & 8 & $(19.5)$ & 0.091 \\
\hline Smoking history, n (\%) & 33 & $(55.0)$ & 7 & $(41.2)$ & 26 & $(63.4)$ & 0.25 \\
\hline \multicolumn{8}{|l|}{ Patients with underlying disease } \\
\hline Diabetes mellitus, n (\%) & 14 & $(23.3)$ & 2 & $(11.8)$ & 12 & $(29.3)$ & 0.31 \\
\hline Bronchial asthma, n (\%) & 12 & $(20.0)$ & 3 & $(17.6)$ & 9 & $(22.0)$ & 1.00 \\
\hline $\mathrm{COPD}^{\mathrm{a}}, \mathrm{n}(\%)$ & 14 & $(23.3)$ & 3 & $(17.6)$ & 11 & $(26.8)$ & 0.74 \\
\hline Liver cirrhosis, n (\%) & 0 & $(0.0)$ & 0 & $(0.0)$ & 0 & $(0.0)$ & - \\
\hline Renal failure, n (\%) & 10 & $(16.7)$ & 2 & $(11.8)$ & 8 & $(19.5)$ & 0.71 \\
\hline Heart failure, n (\%) & 7 & $(11.7)$ & 1 & $(5.9)$ & 6 & $(14.6)$ & 0.66 \\
\hline Malignant neoplasm, n (\%) & 19 & $(31.7)$ & 4 & $(23.5)$ & 15 & $(36.6)$ & 0.54 \\
\hline Patients vaccinated against S. pneumoniae, n (\%) & 13 & $(21.7)$ & 1 & $(5.9)$ & 12 & $(29.3)$ & 0.087 \\
\hline Patients with prior antibiotic therapy, n (\%) & 23 & $(38.3)$ & 4 & $(23.5)$ & 19 & $(46.3)$ & 0.16 \\
\hline Body temperature, ${ }^{\circ} \mathrm{C}$, median (range) & 37.6 & $(35.6-40.2)$ & 37.8 & $(36.3-39.9)$ & 37.6 & $(35.6-40.2)$ & 0.71 \\
\hline White blood cells, $/ \mu \mathrm{L}$, median (range) & 10,850 & $(2000-30,700)$ & 11,900 & $(6100-30,700)$ & 10,700 & $(2000-25,100)$ & 0.52 \\
\hline C-reactive protein, mg/dL, median (range) & 12.23 & $(0.12-40.73)$ & 15.11 & $(0.92-36.7)$ & 12.14 & $(0.12-40.73)$ & 0.046 \\
\hline PORT $^{\mathrm{b}}$ score, points, median (range) & 111.5 & $(32-236)$ & 87 & $(38-236)$ & 124 & $(32-186)$ & 0.10 \\
\hline
\end{tabular}

${ }^{a}$ Chronic obstructive pulmonary disease

${ }^{b}$ Pneumonia Patient Outcomes Research Team 
Table 2 Clinical characteristics of patients with positive results using RAPIRUN with nasopharyngeal swabs

\begin{tabular}{|c|c|c|c|c|}
\hline \multirow[b]{2}{*}{ Age, years, median (range) } & \multicolumn{2}{|c|}{$\begin{array}{l}\text { All pneumococcal } \\
\text { pneumonia patients } \\
(n=17)\end{array}$} & \multicolumn{2}{|c|}{$\begin{array}{l}\text { Patients with positive results using } \\
\text { RAPIRUN with nasopharyngeal swabs } \\
(n=4)\end{array}$} \\
\hline & 78 & $(28-96)$ & 79.5 & $(76-87)$ \\
\hline Male sex, n (\%) & 7 & $(41.2)$ & 0 & $(0.0)$ \\
\hline Nursing home residents, n (\%) & 0 & $(0.0)$ & 0 & $(0.0)$ \\
\hline Smoking history, n (\%) & 7 & $(41.2)$ & 0 & $(0.0)$ \\
\hline \multicolumn{5}{|l|}{ Patients with underlying disease } \\
\hline Diabetes mellitus, n (\%) & 2 & $(11.8)$ & 0 & $(0.0)$ \\
\hline Bronchial asthma, n (\%) & 3 & $(17.6)$ & 0 & $(0.0)$ \\
\hline $\mathrm{COPD}^{\mathrm{a}}, \mathrm{n}(\%)$ & 3 & $(17.6)$ & 0 & $(0.0)$ \\
\hline Liver cirrhosis, n (\%) & 0 & $(0.0)$ & 0 & $(0.0)$ \\
\hline Renal failure, n (\%) & 2 & $(11.8)$ & 0 & $(0.0)$ \\
\hline Heart failure, n (\%) & 1 & $(5.9)$ & 0 & $(0.0)$ \\
\hline Malignant neoplasm, n (\%) & 4 & $(23.5)$ & 1 & $(25.0)$ \\
\hline Patients vaccinated against S. pneumoniae, n (\%) & 1 & $(5.9)$ & 1 & $(25.0)$ \\
\hline Patients with prior antibiotic therapy, n (\%) & 4 & $(23.5)$ & 0 & $(0.0)$ \\
\hline Body temperature, ${ }^{\circ} \mathrm{C}$, median (range) & 37.8 & $(36.3-39.9)$ & 39.1 & $(38.3-39.4)$ \\
\hline White blood cells, / $\mu \mathrm{L}$, median (range) & 11,900 & $(6100-30,700)$ & 9000 & $(6600-13,300)$ \\
\hline C-reactive protein, mg/dL, median (range) & 15.11 & $(0.92-36.7)$ & 17.5 & $(14.1-34.7)$ \\
\hline PORT $^{\mathrm{b}}$ score, points, median (range) & 87 & $(38-236)$ & 92.5 & $(86-101)$ \\
\hline
\end{tabular}

${ }^{a}$ Chronic obstructive pulmonary disease

${ }^{\mathrm{b}}$ Pneumonia Patient Outcomes Research Team

Table 3 Results of sputum culture and antigen detection using urine, sputum, and nasopharyngeal swab samples for 17 patients diagnosed with pneumococcal pneumonia

\begin{tabular}{|c|c|c|c|c|c|}
\hline No. & Sputum culture & Urinary antigen & Sputum antigen & Nasopharyngeal swabs & Nasopharyngeal culture \\
\hline 1 & Streptococcus pneumoniae & - & + & - & S. pneumoniae \\
\hline 2 & Klebsiella pneumoniae & - & + & - & K. pneumoniae \\
\hline 3 & Staphylococcus aureus & + & + & + & S. aureus \\
\hline 4 & Moraxella & + & - & - & a Streptococcus \\
\hline 5 & S. pneumoniae & + & + & - & S. pneumoniae \\
\hline 6 & Neisseria spp & + & - & - & a Streptococcus \\
\hline 7 & S. pneumoniae & + & + & + & S. pneumoniae \\
\hline 8 & S. pneumoniae & - & + & - & a Streptococcus \\
\hline 9 & S. pneumoniae & + & + & + & S. pneumoniae \\
\hline 10 & S. pneumoniae, Escherichia coli & + & + & - & S. aureus \\
\hline 11 & Not detected & + & - & - & S. aureus \\
\hline 12 & S. pneumoniae & + & + & - & a Streptococcus \\
\hline 13 & S. pneumoniae & + & + & - & a Streptococcus \\
\hline 14 & Not detected & + & - & - & S. aureus \\
\hline 15 & S. pneumoniae & + & + & - & a Streptococcus \\
\hline 16 & Not detected & + & - & - & a Streptococcus \\
\hline 17 & S. pneumoniae & + & + & + & a Streptococcus \\
\hline
\end{tabular}


children (under 15 years of age). The results of the present study suggest that RAPIRUN with nasopharyngeal swabs should not be used for the diagnosis of pneumococcal pneumonia in adult patients. The discrepancy of result between RAPIRUN with nasopharyngeal swab and nasopharyngeal culture might suggest that nasopharyngeal swab sample from adult patients with pneumonia are not useful.

In this study, 17 patients were diagnosed with pneumococcal pneumonia using BinaxNOW, RAPIRUN with a sputum sample, or sputum culture. However, these cases may include false positive cases, for example, due to colonization or cross-reaction of antigens, and false negative cases. Because it is very difficult to detect the causative pathogen of pneumonia, clinical data, gram staining of sputum, or radiographic patterns should be considered in addition to sputum culture and rapid antigen detection kits. It might not be suitable to base a diagnosis only on the results of RAPIRUN with a nasopharyngeal swab in adult patients because of low sensitivity.

This study only included inpatients with fairly severe cases of pneumonia. Further studies targeting not only inpatients but also outpatients with pneumonia are required. The small number of pneumococcal pneumonia patients is also a limitation of this study. Further investigation with larger number of participants would be required.

\section{Conclusions}

In conclusion, RAPIRUN exhibited low sensitivity for the diagnosis of pneumococcal pneumonia when used with nasopharyngeal swab samples from adult patients. The causative pathogen of pneumonia should be identified using not only sputum culture or rapid antigen detection kits but also clinical features or gram staining of sputum.

\section{Ethics approval and consent to participate}

The St. Luke's International Hospital Research Ethics Committee approved all aspects of this study (approval number 11-R127, approval date October 24, 2011). Written informed consent was obtained from all participants.

\section{Abbreviations \\ COPD: chronic obstructive pulmonary disease; PORT: Pneumonia Patient Outcomes Research Team.}

\section{Competing interests}

The authors declare that they have no competing interests.

\section{Authors' contributions}

SS contributed to the planning of the study, implementation, data interpretation, and manuscript preparation. NN provided scientific leadership, study implementation, data interpretation, and manuscript preparation. TJ contributed to study design, implementation, data interpretation. YY, Gl, and YT contributed to data collection and study implementation. NU contributed to data analysis and interpretation. NC contributed to directing the study, data collection and study implementation. All authors read and approved the final manuscript.

\section{Acknowledgements}

We thank Ms. Kumiko Soejima for assisting with the statistical analysis.

Funding

The authors declare that there are no sources of funding in this study.

Received: 14 February 2016 Accepted: 21 April 2016

Published online: 20 June 2016

\section{References}

1. WHO. Top 10 causes of death http://www.who.int/gho/mortality_burden_ disease/causes_death/top_10/en/ Accessed 27 April 2016.

2. Welte T, Torres A, Nathwani D. Clinical and economic burden of community-acquired pneumonia among adults in Europe. Thorax. 2012:67(1):71-9.

3. Rozenbaum MH, Pechlivanoglou P, van der Werf TS, Lo-Ten-Foe JR, Postma MJ, Hak E. The role of Streptococcus pneumoniae in community-acquired pneumonia among adults in Europe: a meta-analysis. Eur J Clin Microbiol Infect Dis. 2013;32(3):305-16.

4. Peto L, Nadjm B, Horby P, Ngan TT, van Doorn R, Van Kinh N, et al. The bacterial aetiology of adult community-acquired pneumonia in Asia: a systematic review. Trans R Soc Trop Med Hyg. 2014;108(6):326-37.

5. Cutts FT, Zaman SM, Enwere G, Jaffar S, Levine OS, Okoko JB, et al. Efficacy of nine-valent pneumococcal conjugate vaccine against pneumonia and invasive pneumococcal disease in The Gambia: randomised, double-blind, placebo-controlled trial. Lancet. 2005:365(9465):1139-46.

6. Gutierrez F, Masia M, Rodriguez JC, Ayelo A, Soldan B, Cebrian L, et al. Evaluation of the immunochromatographic Binax NOW assay for detection of Streptococcus pneumoniae urinary antigen in a prospective study of community-acquired pneumonia in Spain. Clin Infect Dis. 2003;36(3):286-92.

7. Dominguez J, Gali N, Blanco S, Pedroso P, Prat C, Matas L, et al. Detection of Streptococcus pneumoniae antigen by a rapid immunochromatographic assay in urine samples. Chest. 2001;119(1):243-9.

8. Horita N, Miyazawa N, Kojima R, Kimura N, Inoue M, Ishigatsubo Y, et al. Sensitivity and specificity of the Streptococcus pneumoniae urinary antigen test for unconcentrated urine from adult patients with pneumonia: a metaanalysis. Respirology. 2013;18(8):1177-83.

9. Ehara N, Fukushima K, Kakeya H, Mukae H, Akamatsu S, Kageyama A, et al. A novel method for rapid detection of Streptococcus pneumoniae antigen in sputum and its application in adult respiratory tract infections. J Med Microbiol. 2008;57(Pt 7):820-6.

10. Izumikawa K, Akamatsu S, Kageyama A, Okada K, Kazuyama Y, Takayanagi N, et al. Evaluation of a rapid immunochromatographic ODK0501 assay for detecting Streptococcus pneumoniae antigen in sputum samples from patients with lower respiratory tract infection. Clin Vaccine Immunol. 2009; 16(5):672-8

11. Mukae H, Yatera K, Noguchi S, Kawanami T, Yamasaki K, Tokuyama S, et al. Evaluation of a rapid immunochromatographic ODK0501 assay for detecting Streptococcus pneumoniae antigens in the sputum of pneumonia patients with positive S. pneumoniae urinary antigens. J Infect Chemother. 2015;21(3): 176-81.

12. Iwata S, Matsubara K, Kawamura N, Shimizu K, Azumagawa K, Morinobu T, et al. Clinical performance evaluation of Streptococcus pneumoniae antigen detection kit ODK0501 in childhood respiratory tract infections. J Pediatr Infect Dis Immunol. 2011;23(1):3-9.

13. Barrett-Connor $E$. The nonvalue of sputum culture in the diagnosis of pneumococcal pneumonia. Am Rev Respir Dis. 1971;103(6):845-8.

14. Lentino JR, Lucks DA. Nonvalue of sputum culture in the management of lower respiratory tract infections. J Clin Microbiol. 1987:25(5):758-62. 